\title{
Variación interpoblacional y ontogenética en la dieta de la rana llorona Physalaemus albonotatus (Anura: Leiuperidae)
}

\section{Interpopulation and ontogenetic variation in the diet of the menwig frog Physalaemus albonotatus (Anura: Leiuperidae)}

\author{
Diego Alejandro Falico ${ }^{1,2 凶}$, Javier Alejandro López ${ }^{1,3}$, Carolina Elizabet Antoniazzi ${ }^{1,3}$ y Adolfo Héctor Beltzer ${ }^{1,2}$ \\ Instituto Nacional de Limnología (CONICET-UNL). Ciudad Universitaria. Paraje el Pozo (3000) Santa Fe, Argentina. \\ ${ }^{2}$ Universidad Autónoma de Entre Ríos, Facultad de Ciencia y Tecnología. Andrés Pazos y Corrientes (3100) Paraná, Argentina. \\ ${ }^{3}$ Universidad Nacional del Litoral, Facultad de Humanidades y Ciencias, Departamento de Ciencias Naturales. Ciudad Universitaria. Paraje el Pozo \\ (3000) Santa Fe, Argentina. \\ $\triangle$ dialefalico@gmail.com
}

\begin{abstract}
Resumen. Se analizó y comparó el espectro trófico de 2 poblaciones de Physalaemus albonotatus que habitan en ecosistemas diferentes: a) un terreno baldío urbano y b) un área protegida del valle aluvial del río Paraná Medio. La dieta de Physalaemus albonotatus está compuesta fundamentalmente de hormigas, coleópteros, colémbolos e isópodos, pero con numerosas presas secundarias. Al comparar 3 grupos etarios de los individuos en cada población (adultos, subadultos y jóvenes) se encontró una variación ontogenética relacionada con el volumen, cantidad y tipo de presa ingerida. Los anfibios adultos consumieron mayor cantidad de presas y más voluminosas, pero el nicho trófico de los jóvenes fue más amplio. Entre los adultos, la similitud de la dieta entre sexos fue muy elevada, aunque las hembras consumieron más hormigas que los machos. Si bien el solapamiento trófico entre las poblaciones fue elevado, la amplitud trófica resultó mayor en la población del humedal fluvial. Las diferencias en la alimentación encontradas entre las 2 poblaciones y lo registrado en otros trabajos realizados en diferentes ambientes reflejan la plasticidad trófica de este anuro, característica que le permite prosperar en hábitats diversos, incluso en los muy antropizados.
\end{abstract}

Palabras clave: anuros, hábitats de forrajeo, ambiente urbano, humedal fluvial, plasticidad trófica, solapamiento trófico.

\begin{abstract}
We analyzed and compared the feeding of 2 populations of Physalaemus albonotatus inhabiting different ecosystems: a) an urban vacant lot, and b) a protected area of Middle Paraná River floodplain. The diet of Physalaemus albonotatus is mainly composed by ants, beetles, springtails and woodlouses, but also included several secondary preys. While comparing adults, subadults and juveniles diet on each population, we found an ontogenetic diet variation related to changes in prey type and volume as well as in number of prey items per gut. Bigger frogs consumed a greater amount of prey and more voluminous arthropods, while juveniles had a larger trophic niche breadth. Despite the great similarity in diet between sexes, adult females consumed a larger amount of ants than males did. Although trophic niche overlap between both populations was high, trophic niche breadth was wider for Paraná River floodplain population. Feeding differences found between studied populations and populations inhabiting other environments as described on literature reflects the trophic plasticity of this species and its capacity to thrive at different habitats, including markedly anthropogenically impacted environments.
\end{abstract}

Key words: anurans, foraging habitat, urban environment, floodplain wetland, trophic plasticity, trophic overlap.

\section{Introducción}

Los humedales de Sudamérica sustentan altos valores de biodiversidad (Neiff, 1996), pero durante los últimos 100 años han sido gradualmente destruidos y utilizados para la agricultura y/o considerados como impedimento para el progreso y reservorio potencial de enfermedades (Brinson y Malvárez, 2002). Una de las principales causas de la pérdida de biodiversidad y particularmente de la

Recibido: 20 octubre 2011; aceptado: 04 mayo 2012 declinación de anfibios, es la degradación y destrucción de ecosistemas naturales (Alford y Richards, 1999). Este fenómeno mundial también ha sido reconocido en los ambientes del río Paraná medio (Peltzer y Lajmanovich, 2001). En este marco, la capacidad de las especies para prosperar en diferentes ambientes podría resultar útil para mejorar su posibilidad de supervivencia en un ambiente cambiante.

La alimentación, el tiempo y el lugar donde esta actividad se realiza componen una de las principales dimensiones del nicho ecológico (Schoener, 1974). La 
información sobre la ecología alimentaria es crucial para entender el impacto de las modificaciones de los hábitats sobre los anfibios (Anderson et al., 1999), así como para evaluar si las distintas especies de anuros ajustan sus dietas a los distintos hábitats que ocupan, de acuerdo con la variación en la disponibilidad de las diferentes presas (Peltzer et al., 2010). Existe evidencia de que la alimentación de los anfibios depende del microhábitat de forrajeo (Duré y Kehr, 2004) y que puede variar entre poblaciones que habitan ambientes diferentes (e.g., López et al., 2005a; Peltzer et al., 2010). Sin embargo, los estudios sobre alimentación generalmente se enfocan en un determinado ambiente dentro de la diversidad de hábitats que utiliza cada especie (e.g., Lajmanovich, 1996; Hirai y Matsui, 1999; 2002; López et al., 2007; 2009). Además, son pocos los estudios donde se analiza la variación ontogénica de la dieta (e.g., Lajmanovich, 1996; Hirai, 2002; López et al., 2007; Quiroga et al., 2009). Esta situación motivó la presente investigación para determinar la variación interpoblacional y ontogenética de la alimentación de Physalaemus albonotatus, que permitirá comprender mejor la relación de este anuro con el ambiente donde habita, su respuesta a la disponibilidad de presas en cada tipo de ambiente y las posibilidades de prosperar en sitios con diferente grado de perturbación antrópica.

\section{Materiales y métodos}

Área de estudio. Los muestreos se realizaron entre octubre de 2004 y marzo de 2005 en 2 ecosistemas diferentes de la Provincia de Santa Fe, Argentina: a) la Reserva de la Universidad Nacional del Litoral de la ciudad de Santa Fe (R-UNL; 31 38'12.34" S, 6040'22.32” O) y b) un terreno baldío de la ciudad de Santo Tomé denominado "Cancha Don Sebastián" (ST; 3140’17.50” S, 6046’17.66" O) (Fig. 1).

La R-UNL es una fracción de aproximadamente 6 ha de los hidrosistemas que componen el valle aluvial del río Paraná (Gagneten et al., 2000), que corresponde a la ecorregión Delta e Islas del Paraná (Brown et al., 2006). La R-UNL posee una laguna permanente en su parte central, charcas temporales, un albardón perimetral discontinuo y algunos albardones interiores aislados dominados por un bosque en galería. Durante los muestreos, las condiciones climáticas oscilaron entre $19-27^{\circ} \mathrm{C}$ de temperatura (T), $62-75 \%$ de humedad relativa (HR) y $66-353 \mathrm{~mm}$ de precipitación mensual acumulada $(\mathrm{P})$. Este ambiente se encuentra protegido, pero no se halla exento de perturbaciones antrópicas, principalmente vinculadas con la dinámica de sus cuerpos de agua. Por otro lado, ST es un descampado urbano en la ciudad de Santo Tomé que, si bien se encuentra cercano a la R-UNL (10.5 km en línea recta;
Fig. 1), corresponde a la ecorregión del Espinal (Brown et al., 2006). Este predio abarca un área aproximada de 2.5 ha, sin un cuerpo de agua permanente, pero con zonas bajas que se anegan frecuentemente tras las lluvias y donde se registró la presencia de vegetación palustre. Las condiciones climáticas para este sitio oscilaron entre T: $18^{\circ}-26^{\circ}$ C, HR: 69-73\%, P: 53-403 milímetros.

Captura de los ejemplares y análisis de los contenidos gastrointestinales. Los anfibios se capturaron con trampas de caída (Heyer et al., 1994) y se ingresaron a la colección herpetológica de referencia del Laboratorio de Herpetología del Instituto Nacional de Limnología (CONICET-UNL). Se registró la longitud hocico-cloaca (LHC) de cada ejemplar con un calibre digital milimétrico de precisión $0.01 \mathrm{~mm}$. El peso de cada individuo $(\mathrm{P})$ fue estimado con una balanza digital de precisión 0.01 gr. Se extrajeron los tractos gastrointestinales y los contenidos se determinaron taxonómicamente hasta el nivel de orden o familia en la mayoría de los casos. El volumen de las presas (V) se estimó mediante la fórmula del esferoide ensanchado (Dunham, 1983): $\mathrm{V}=4 / 3 \pi(\mathrm{L} / 2)(\mathrm{A} / 2)^{2}$ en $\mathrm{mm}^{3}$; donde $\mathrm{L}=$ largo de la presa y $\mathrm{A}=$ ancho máximo de la presa.

Análisis numérico y estadístico. Para relacionar el tamaño $(\mathrm{LHC})$ y peso $(\mathrm{P})$ de los ejemplares con el número $(\mathrm{N})$ y volumen $(\mathrm{V})$ de las presas ingeridas se utilizaron correlaciones de Spearman, debido al incumplimiento del requisito de normalidad requerido para la utilización de análisis paramétricos. Para calcular la diversidad trófica se usó el método propuesto por Pielou (1969): $1-D=1-[(\Sigma$ $\left.\stackrel{\mathrm{S}}{\mathrm{i}=1} n_{i}\left(n_{i}-1\right)\right] /[N(N-1)]$; donde $S$ es el número de especies (i.e., presa), $N$ es el total de organismos presentes y $n$ es el número de ejemplares por especie (i.e., presa). Se calculó la diversidad media $(\mathrm{H})$ y la diversidad trófica acumulada (Hk) que permite determinar la muestra mínima,

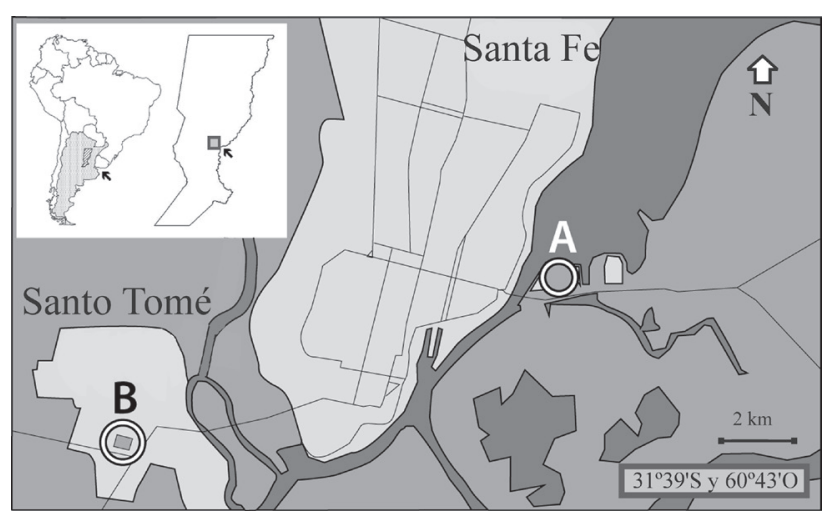

Figura 1. Ubicación de los sitios de muestreo. A, Reserva de la Universidad Nacional del Litoral dentro del valle aluvial del río Paraná, y B, terreno baldío dentro del ejido urbano de la ciudad de Santo Tomé. 
según la siguiente fórmula (Hurtubia, 1973): $\mathrm{Hk}=(\mathrm{Nk} *$ $\mathrm{Hk}-\mathrm{Nk}-1$ * Hk-1) / (Nk - Hk-1); donde Hk y Hk-1 son las diversidades tróficas acumuladas en $\mathrm{k}$ y k-1 tractos gastrointestinales y Nk y Nk-1 son el número total de individuos de todas las especies presa en $\mathrm{k} \mathrm{y} \mathrm{k-1}$ tracto digestivo. La amplitud del nicho trófico se obtuvo mediante el índice de Levins (1968): $\mathrm{Nb}=\left(\sum \mathrm{P}_{i j}{ }^{2}\right)^{-1}$; donde $\mathrm{P}_{i j}$ es la probabilidad de la proporción de la presa $i$ en la muestra $j$.

Para ponderar la contribución de cada categoría de alimento a la dieta se calculó el índice de importancia relativa jerarquizado (IRI\%) (George y Hadley, 1979): $\mathrm{IRI} \%=(100 * \mathrm{AL}) / \Sigma \mathrm{AL}$; donde $\mathrm{AL}=$ frecuencia porcentual de la presencia de la presa (proporción de la numerosidad representada por la presa + proporción del volumen representado por la misma). No se analizaron las diferencias entre sexos en la población de ST debido a que sólo se contó con 2 ejemplares adultos. Para el análisis del solapamiento del nicho trófico entre las poblaciones y grupos etarios se usó el índice simétrico propuesto por Pianka (1974), que responde a la formula: $\mathrm{O}_{\mathrm{jk}}=\sum \mathrm{P}_{i j} * \mathrm{P}_{i k} / \sqrt{ }\left(\sum \mathrm{P}_{i j}{ }^{2}\right.$ $\left.* \sum \mathrm{P}_{i k}{ }^{2}\right)$; donde $\mathrm{P}_{i j}$ y $\mathrm{P}_{i k}$ representan las proporciones del recurso $i$ utilizado por las especies $j$ y $k$ respectivamente. Este índice genera valores entre 0 (sin solapamiento) y 1 (máximo solapamiento).

\section{Resultados}

Se analizaron 128 tractos digestivos de $P$. albonotatus (R-UNL: $\mathrm{n}=82$ : ST: $\mathrm{n}=46$ ). Se contabilizaron 1020 presas, 733 en los estómagos (71.85\%) (R-UNL $n=445$; ST $n=288)$ y 287 en los intestinos (28.15\%) (R-UNL $n=$ 184; ST $n=103)$. Estas presas fueron clasificadas en 21 categorías taxonómicas.

Las correlaciones estadísticamente significativas $(p<$ 0.05 ) entre el peso (P) y tamaño (LHC) de los ejemplares y el número $(\mathrm{N})$ y volumen $(\mathrm{V})$ de las presas ingeridas para la población de R-UNL fueron: $\mathrm{P} v s . \mathrm{N}(\mathrm{r}=0.3174$, $p=0.0074), \mathrm{P} v s . \mathrm{V}(\mathrm{r}=0.4618, p<0.0001), \mathrm{LHC} v s . \mathrm{N}(\mathrm{r}=$ $0.3251, p=0.006)$ y LHC $v s$. V $(\mathrm{r}=0.4778, p<0.0001)$. Mientras que para ST sólo resultó significativa la correlación entre $\mathrm{P} v s . \mathrm{V}, \mathrm{r}=0.3569, p=0.0238$; en tanto LHC vs. $\mathrm{V}(\mathrm{r}=0.2981, p=0.0617)$ fue marginalmente no significativa y no significativa para $\mathrm{P} v s . \mathrm{N}(\mathrm{r}=0.2086$; $p=$ $0.1964)$.

Los valores de diversidad trófica para la población de R-UNL fueron: $\mathrm{H}_{\text {total }}=0.74, \mathrm{H}_{\text {adultos }}=0.72, \mathrm{H}_{\text {subadultos }}=$ $0.72 \mathrm{y} \mathrm{H}_{\mathrm{jóvenes}}=0.81$. La estabilización de las curvas de la diversidad trófica acumulada se obtuvo en los tractos digestivos $17,12,5$ y 14 para el total de la población, los adultos, los subadultos y los jóvenes, respectivamente. Para los adultos, la diversidad trófica de los machos fue mayor que la de las hembras $\left(\mathrm{H}_{\phi}=0.81 \mathrm{y} \mathrm{H}_{\varphi}=0.74\right)$. Las muestras mínimas estimadas para estos 2 grupos fueron 10 y 8 tractos digestivos respectivamente. Los valores de diversidad trófica en ST fueron: $\mathrm{H}_{\text {total }}=0.86 ; \mathrm{H}_{\text {subadul- }}$ $\mathrm{tos}_{\mathrm{t}}=0.92 \mathrm{y} \mathrm{H}_{\mathrm{jovenes}}=0.84$. Las curvas de diversidad trófica acumulada del total de la población y para los subadultos se estabilizan en los tractos digestivos 14 y 3, respectivamente; mientras que para jóvenes la curva no alcanzó una estabilización que indique que se haya alcanzado la muestra mínima.

La amplitud de nicho trófico en R-UNL sin discriminar, por grupo etario, fue: $\mathrm{Nb}_{\mathrm{R}-\mathrm{UNL}}=1.06$. Este valor fue mayor mientras más jóvenes resultaban los ejemplares $\left(\mathrm{Nb}_{\text {adultos }}=0.69 ; \mathrm{Nb}_{\text {subadulto }}=0.71 ; \mathrm{Nb}_{\text {jóvenes }}=1.66\right)$. Las hembras adultas tuvieron una amplitud de nicho trófico menor que los machos adultos $\left(\mathrm{Nb}_{0}=0.6\right.$ y $\left.\mathrm{Nb}_{8}=0.77\right)$. La amplitud de nicho trófico en ST sin discriminar por grupo etario resultó más baja que la de la población de R-UNL $\left(\mathrm{Nb}_{\mathrm{ST}}=0.78\right)$. Para adultos se registró el valor más bajo $(\mathrm{Nb}=0.2)$, pero este valor puede deberse a que el cálculo se realizó para los únicos 2 ejemplares capturados de este grupo etario en ST. Al igual que en la población de R-UNL, la amplitud de nicho de los subadultos fue menor que la de los jóvenes $\left(\mathrm{Nb}_{\text {subadultos }}=0.63 \mathrm{y} \mathrm{Nb}_{\text {jóvenes }}=1.18\right)$.

La frecuencia y el número de hormigas resultaron elevados en la dieta de las ranas de R-UNL. Asimismo, los coleópteros resultaron frecuentes y numerosos en la alimentación de las ranas de la población ST (Cuadro 1).

Según el índice jerarquizado de importancia relativa de las presas (IRI\%), las hormigas resultaron el grupo más importante para la dieta de los ejemplares de la población de R-UNL: IRI $\%_{\text {total }}=59.5$; IRI $\%_{\text {adultos } 2}=44.95$; IRI $\%_{\text {adultos } 9}=59.54 ;$ IRI $\%_{\text {subadultos }}=65.68 \%$; IRI $\% \%_{\text {jóvenes }}=$ $59.22 \%$; seguido por isópodos: IRI $\%_{\text {total }}=23.25$; IRI $\%_{\text {adultos } 8}=28.43 ; \quad$ IRI $\%_{\text {adultos } 9}=15.53 ; \quad$ IRI $\%_{\text {subadultos }}=$ $27.78 ;$ IRI $\%_{\text {jóvenes }}=21.14$. Los organismos que ocupan el tercer y cuarto lugar de importancia variaron entre grupos etarios; en el tercero, los coleópteros para: IRI $\%_{\text {total }}=$ $8.13 ; \mathrm{IRI} \%_{\text {adultos } \delta}=14 ; \mathrm{IRI} \%_{\text {adultos } \bigcirc}=15.67 ; \mathrm{IRI} \%_{\text {Subadultos }}=$ 2.53 y los colémbolos: IRI $\%$ ávenes $=5.63$, y en el cuarto, las arañas: IRI $\%_{\text {total }}=4.05$; IRI\% $\%_{\text {adultos } \delta}=10.03$; IRI $\%_{\text {adultos } 9}=$ 6.48 ; los colémbolos: IRI $\%_{\text {subadultos }}=2.04$ y los coleópteros: $\mathrm{IRI} \%_{\text {jóvenes }}=4.36$ (Fig. 2 ).

En la población de ST, el primer y segundo lugar en el orden de importancia relativa de las presas en la dieta de los diferentes grupos etarios se repartió entre coleópteros y colémbolos. El primer lugar de importancia fue ocupado por coleópteros para: IRI $\%_{\text {total }}=47.18$; IRI $\%_{\text {subdultos }}=60.71$ y por colémbolos para: IRI $\%_{\text {jóvene }}=$ 47.1. Los colémbolos se ubicaron en segundo lugar en: IRI $\%_{\text {total }}=22.14 ;$ IRI $\%_{\text {subadultos }}=11.09$ y los coleópteros en: IRI $\%_{\text {jóvenes }}=32.88$. El tercer lugar de importancia estuvo ocupado por dípteros: IRI $\%_{\text {total }}=14.10 ;$ IRI $\%_{\text {jóvenes }}=9.94$ 
Cuadro 1. Frecuencia de la presencia de las presas $(F)$ y número de las consumidas $(N)$ por las ranas de la Reserva Universitaria (R-UNL) y del descampado urbano de la ciudad de Santo Tomé (ST) *

\begin{tabular}{|c|c|c|c|c|c|c|c|c|c|c|c|c|c|c|c|c|c|c|c|c|}
\hline & \multicolumn{12}{|c|}{$R-U N L$} & \multicolumn{8}{|c|}{$S T$} \\
\hline & \multicolumn{6}{|c|}{$F$} & \multicolumn{6}{|c|}{$N$} & \multicolumn{4}{|c|}{$F$} & \multicolumn{4}{|c|}{$N$} \\
\hline & $T$ & $A$ & $S$ & $J$ & $H$ & $M$ & $T$ & $A$ & $S$ & $J$ & $H$ & $M$ & $T$ & $A$ & $S$ & $J$ & $T$ & A & $S$ & $J$ \\
\hline \multicolumn{21}{|l|}{ INSECTA } \\
\hline Collembola & 18 & 4 & 4 & 10 & 2 & 2 & 52 & 11 & 15 & 26 & 7 & 4 & 20 & 0 & 12 & 8 & 110 & 0 & 26 & 88 \\
\hline Thysanoptera & 4 & 1 & 0 & 3 & 1 & 0 & 6 & 1 & 0 & 5 & 1 & 0 & 0 & 0 & 0 & 0 & 0 & 0 & 0 & 0 \\
\hline Ephemeroptera & 0 & 0 & 0 & 0 & 0 & 0 & 0 & 0 & 0 & 0 & 0 & 0 & 0 & 0 & 0 & 0 & 0 & 0 & 0 & 0 \\
\hline Homoptera & 4 & 0 & 1 & 3 & 0 & 0 & 10 & 0 & 1 & 9 & 0 & 0 & 0 & 0 & 0 & 0 & 0 & 0 & 0 & 0 \\
\hline Hemiptera & 4 & 2 & 1 & 1 & 1 & 1 & 4 & 2 & 1 & 1 & 1 & 1 & 8 & 0 & 6 & 2 & 11 & 0 & 7 & 4 \\
\hline Larv. Hemiptero & 1 & 1 & 0 & 0 & 1 & 0 & 1 & 1 & 0 & 0 & 1 & 0 & 1 & 0 & 1 & 0 & 1 & 0 & 1 & 0 \\
\hline Lepidoptera & 1 & 1 & 0 & 0 & 0 & 1 & 1 & 1 & 0 & 0 & 0 & 1 & 0 & 0 & 0 & 0 & 0 & 0 & 0 & 0 \\
\hline Larv. Lepidoptera & 1 & 0 & 0 & 1 & 0 & 0 & 1 & 0 & 0 & 1 & 0 & 0 & 1 & 0 & 1 & 0 & 2 & 0 & 2 & 0 \\
\hline Coleoptera & 28 & 14 & 4 & 10 & 7 & 7 & 66 & 48 & 5 & 13 & 30 & 18 & 29 & 2 & 18 & 9 & 83 & 4 & 56 & 23 \\
\hline Larv. Coleoptero & 3 & 1 & 1 & 1 & 1 & 0 & 3 & 1 & 1 & 1 & 0 & 1 & 1 & 0 & 0 & 1 & 4 & 0 & 2 & 2 \\
\hline Hymenoptera & 12 & 4 & 3 & 5 & 3 & 1 & 22 & 7 & 3 & 12 & 4 & 3 & 5 & 0 & 3 & 2 & 77 & 6 & 56 & 15 \\
\hline Larv. Hymenoptera & 1 & 0 & 0 & 1 & 0 & 0 & 1 & 0 & 0 & 1 & 0 & 0 & 0 & 0 & 0 & 0 & 0 & 0 & 0 & 0 \\
\hline Formicidae & 59 & 23 & 13 & 23 & 12 & 11 & 320 & 143 & 88 & 89 & 100 & 43 & 23 & 2 & 16 & 5 & 10 & 4 & 3 & 3 \\
\hline Diptera & 4 & 2 & 1 & 1 & 2 & 0 & 4 & 2 & 1 & 1 & 2 & 0 & 20 & 2 & 10 & 8 & 26 & 5 & 11 & 10 \\
\hline Larv. Diptera & 2 & 1 & 0 & 1 & 0 & 1 & 2 & 1 & 0 & 1 & 0 & 1 & 1 & 0 & 1 & 0 & 2 & 0 & 1 & 1 \\
\hline Blattodea & 1 & 0 & 1 & 0 & 0 & 0 & 1 & 0 & 1 & 0 & 0 & 0 & 0 & 0 & 0 & 0 & 0 & 0 & 0 & 0 \\
\hline \multicolumn{21}{|l|}{ ARACHNIDA } \\
\hline Araneae & 20 & 11 & 3 & 6 & 5 & 6 & 26 & 14 & 3 & 9 & 7 & 7 & 7 & 2 & 2 & 3 & 11 & 5 & 3 & 3 \\
\hline Acari & 10 & 2 & 2 & 6 & 2 & 0 & 14 & 3 & 4 & 7 & 3 & 0 & 13 & 2 & 8 & 3 & 19 & 2 & 9 & 8 \\
\hline Pseudoescorpionidae & 13 & 4 & 1 & 8 & 2 & 2 & 15 & 4 & 1 & 10 & 2 & 2 & 0 & 0 & 0 & 0 & 0 & 0 & 0 & 0 \\
\hline Opilionidae & 0 & 0 & 0 & 0 & 0 & 0 & 0 & 0 & 0 & 0 & 0 & 0 & 13 & 0 & 10 & 3 & 20 & 0 & 15 & 5 \\
\hline \multicolumn{21}{|l|}{ CRUSTACEA } \\
\hline Isopoda & 29 & 12 & 7 & 10 & 6 & 6 & 79 & 25 & 29 & 25 & 10 & 15 & 7 & 0 & 5 & 2 & 0 & 0 & 0 & 0 \\
\hline \multicolumn{21}{|l|}{ MIRIAPODA } \\
\hline Diplopoda & 1 & 1 & 0 & 0 & 1 & 0 & 1 & 1 & 0 & 0 & 1 & 0 & 0 & 0 & 0 & 0 & 0 & 0 & 0 & 0 \\
\hline
\end{tabular}

*Abreviaturas de las columnas: T, toda la población; A, adultos; S, subadultos; J, jóvenes; H, hembras adultas; M, machos adultos; Larv., larvas.

e himenópteros no formícidos: IRI $\%_{\text {subadultos }}=7.61$. En cuarto lugar se encontraron himenópteros no formícidos: IRI $\%_{\text {total }}=5.28 ;$ IRI $\%_{\text {jóvenes }}=2.91$ y dípteros IRI $\%_{\text {subadultos }}=$ 7.08 (Fig. 2).

El solapamiento trófico entre las 2 poblaciones sin discriminar por grupo etario fue: $\mathrm{O}_{\mathrm{jk}}=0.69$. Los subadultos presentaron un solapamiento mayor $\left(\mathrm{O}_{\mathrm{jk}}=0.73\right)$, mientras que en los jóvenes fue menor: $\mathrm{O}_{\mathrm{jk}}=0.46$. El análisis de solapamiento entre adultos de las 2 poblaciones no fue posible debido al escaso número de ejemplares adultos analizados para ST. Entre machos y hembras de R-UNL el solapamiento fue elevado: $\mathrm{O}_{\mathrm{jk}}=0.97$.

\section{Discusión}

El análisis de solapamiento trófico en este estudio mostró que las 2 poblaciones de $P$. albonotatus tienen diferencias en la importancia de las presas que consumen según el ambiente donde habitan; esta diferencia resultó sustancialmente mayor entre los ejemplares de menor 

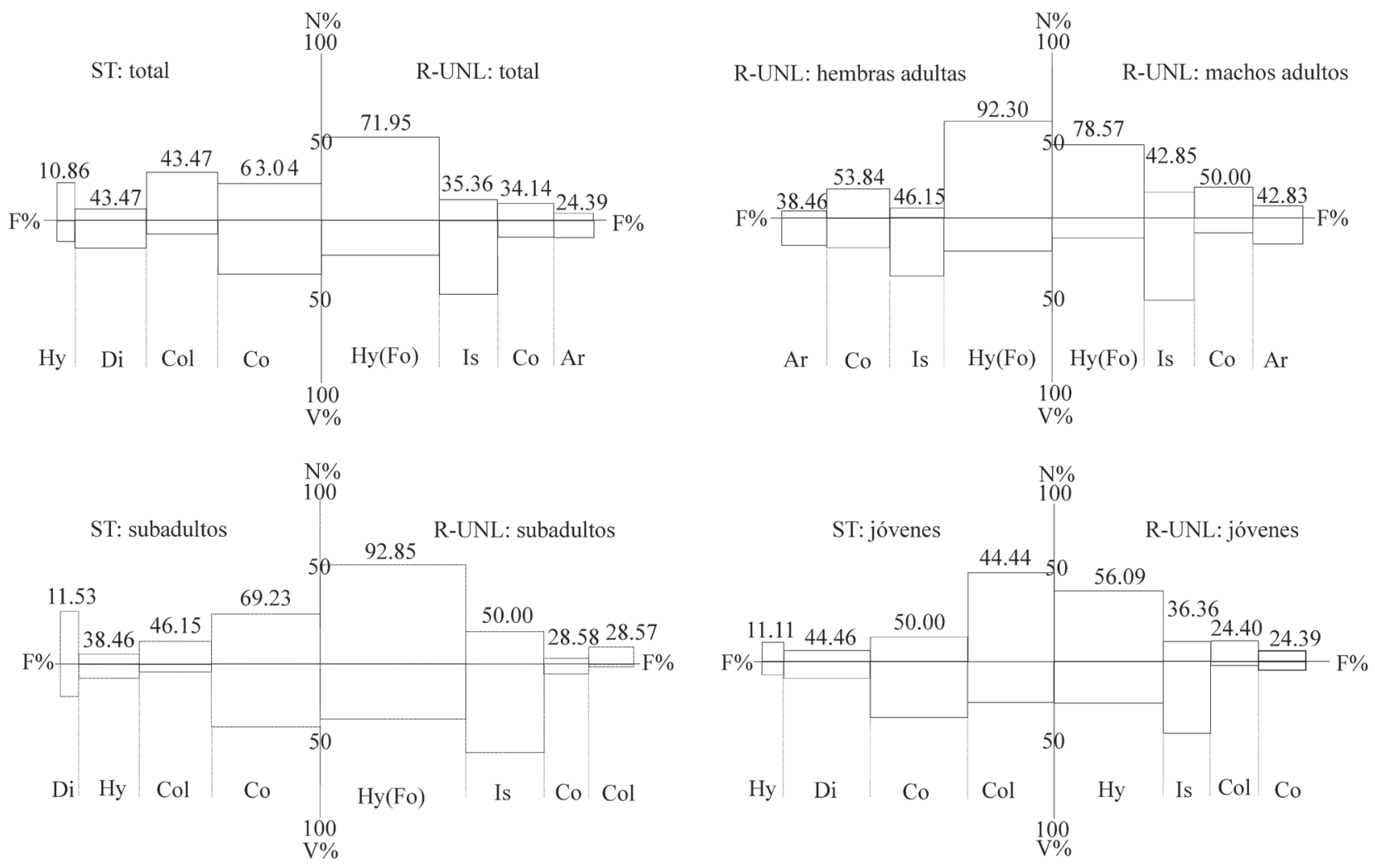

Figura 2. Índice de Importancia Relativa de las presas consumidas por las ranas del descampado urbano de la ciudad de Santo Tomé (ST) y de la Reserva Universitaria (R-UNL), representado para el total de la población sin discriminar por grupo etario, para los machos y hembras adultas de la R-UNL y para los subadultos y jóvenes de cada población. F\%, frecuencia de la presencia porcentual de la presa; N\%, proporción de la abundancia (cantidad de ejemplares) representada por la misma y V\%, proporción de su volumen. Ar, Araneae; Co, Coleoptera; Col, Collembola; Di, Diptera; Hy(Fo), Hymenoptera, Formicidae; Hy, Hymenoptera no Formicidae; Is, Isopoda.

tamaño. Se ha demostrado que la disponibilidad de presas es uno de los factores más importantes en la determinación de la dieta de los anfibios (Hirai y Matsui, 1999; 2002; López et al., 2009), por lo que las diferencias encontradas entre las 2 poblaciones probablemente se relacionan con diferencias en la presencia y abundancia de los tipos de presas consumidas (Levings, 1983).

En la dieta de $P$. albonotatus puede observarse una variación según sus poblaciones se encuentren habitando ambientes tan disímiles como campos cultivados, bosques nativos conservados o degradados, bosques fluviales o pastizales y pajonales (este estudio; López et al., 2005b; Peltzer et al., 2010). Un patrón que se repite, tanto en las 2 poblaciones estudiadas como en poblaciones analizadas en trabajos anteriores (López et al., 2005b; Peltzer et al., 2010), es que más allá del cambio en la composición de la dieta de $P$. albonotatus siempre existen 1 o 2 grupos alimenticios dominantes (e.g., formícidos e isópodos en R-UNL y coleópteros y colémbolos en ST) sobre un número elevado de presas secundarias, marcadamente más accesorias. Además, los organismos más importantes en la alimentación de estos leiuperidos resultan ser casi siempre pequeños artrópodos terrestres caminadores y en la mayoría de los casos son insectos gregarios que se encuentran en parches de alta densidad dentro de la heterogeneidad del microhábitat de forrajeo (este estudio; López et al., 2005b; Peltzer et al., 2010). Es probable que la incorporación de una $\mathrm{u}$ otra presa ecomorfológicamente similar (e.g., insectos marchadores terrestres y quitinosos; por ejemplo, hormigas $v s$. termitas o pequeños coléopteros) responda en principio a su disponibilidad y abundancia relativa en el ambiente (López et al., 2007).

La mayor amplitud del nicho trófico en hembras puede estar relacionada con una variación en la estrategia de forrajeo debida a los requerimientos energéticos y nutricionales propios de la gran inversión para el desarrollo y maduración gonadal (Duellman y Trueb, 1986; Wells, 2007). En tanto, los cambios ontogenéticos encontrados 
sugieren que las poblaciones de anfibios deben ser abordadas como un conjunto de subpoblaciones definidas por grupos etarios (según la talla), ya que el cambio estacional de la estructura de tamaños de la población debido a la incorporación y crecimiento de las nuevas cohortes (López et al., 2011) hace que su impacto como depredadores (y probablemente como presas) en los ecosistemas varíe estacionalmente. En este sentido, la menor similitud en la dieta de los jóvenes de las 2 poblaciones estudiadas y su mayor amplitud dietaria respecto a los subadultos y adultos indican un elevado oportunismo depredador por parte de los ejemplares recién metamorfoseados y la asociada alimentación generalista (Feder y Burggren, 1992). Por otro lado, las correlaciones entre el tamaño de las presas y los anuros muestran que existen restricciones inherentes al grupo etario al momento de seleccionar las presas (Feder y Burggren, 1992; López et al., 2007). Si bien este patrón de variación ontogenética en la amplitud trófica se comparte con otras especies de anfibios (Lajmanovich, 1996; Hirai, 2002), no resulta universal (López et al., 2007), por lo que es necesario incrementar los estudios de variación ontogenética de la dieta en diferentes especies para poder realizar generalizaciones fundadas al respecto.

Refiriéndose a las estrategias tróficas, Williams et al. (2006) señalaron que las especies generalistas deben ser menos susceptibles a las fluctuaciones en cualquier tipo de alimento particular que las especies que se alimentan de manera más especializada. En tanto, la teoría del forrajeo óptimo sugiere que la especialización en la dieta puede ocurrir cuando la abundancia de ciertos recursos tróficos se encuentra constantemente elevada (Pyke, 1984). Al menos en la reserva universitaria (R-UNL), las hormigas representan el segundo grupo de presas más abundantes, sólo superado por la disponibilidad de colémbolos (López et al., 2007) y fueron las principales presas en la alimentación de la población de P. albonotatus de R-UNL. Una dieta diversa e incluso más amplia en el caso de los jóvenes, pero con cierta tendencia al consumo de los artrópodos abundantes en la oferta trófica, podría permitir a P. albonotatus sortear los cambios producidos en la disponibilidad ambiental de presas producto de la alteración ambiental. Esta plasticidad trófica sería una de las claves que permite a P. albonotatus prosperar en ecosistemas marcadamente diferentes e incluso con distintos grados de antropización.

\section{Agradecimientos}

Los autores agradecen a Romina Ghirardi y Pablo A. Scarabotti por su colaboración en los muestreos y distintas etapas de la realización del presente trabajo. A José Paez por su colaboración en la elaboración de la figura del área de estudio. A los revisores anónimos que contribuyeron a mejorar el contenido del manuscrito. El presente trabajo forma parte de la tesis de licenciatura de Diego A. Falico (FCyT, UADER).

\section{Literatura citada}

Alford, R. A. y S. J. Richards. 1999. Global amphibian declines: a problem in applied ecology. Annual Review of Ecology, Evolution and Systematics 30:133-165.

Anderson, A. M., D. A. Haukos y J. T. Anderson. 1999. Diet composition of three anurans from the Playa wetlands of northwest Texas. Copeia 2:515-520.

Brinson, M. M. y A. I. Malvárez. 2002. Temperate freshwater wetlands: types, status, and threats. Environmental Conservation 29:115-133.

Brown, A. D., U. Martínez-Ortiz, M. Acerbi y J. Corchera. (eds.). 2006. La situación ambiental de la Argentina 2005. Fundación Vida Silvestre Argentina, Buenos Aires. 587 p.

Duellman, W. E y L. Trueb. 1986. Biology of Amphibians. The Johns Hopkins University Press. Baltimore. 670 p.

Dunham, A. E. 1983. Realized niche overlap, resource abundance and intensity of interspecific competition. In Lizard Ecology, R. B. Huey, E. R. Pianka y T. W. Shoener (eds.). Harvard University Press, Cambridge, Massachusetts. p. 261-280.

Duré, M. I. y A. I. Kehr. 2004. Influence of microhabitat on the trophic ecology of two leptodactylids from northeastern Argentina. Herpetologica 60:295-303.

Feder, M. E. y W. W. Burggren. 1992. Environmental physiology of the amphibians University of Chicago Press, Chicago. 646 p.

Gagneten, A. M., A. L. Ronchi, F. Rojas-Molina y R. Sobrero. 2000. Aportes al conocimiento del ambiente acuático de la Reserva Ecológica de la Ciudad Universitaria "El Pozo" y de su diversidad zooplanctónica. Revista FABICIB 4:111-122.

George, E. L. y W. F. Hadley. 1979. Food and habitat partitioning between rock bass (Ambloplites rupestris) and smallmouth bass (Micropterus dolomieui) young of the year. Transactions of the American Fisheries Society 108:253-261.

Heyer, W., M. Donnelly, R. McDiarmind, L. C. Hayek y M. Foster. 1994. Measuring and monitoring biological diversity. Standard methods for amphibians. Smithsonian Institution, Washington, D.C. 364 p.

Hirai, T. 2002. Ontogenetic change in the diet of the pond frog, Rana nigromaculata. Ecological Research 17:639-634.

Hirai, T. y M. Matsui. 1999. Feeding habits of the pond frog, Rana nigromaculata, inhabiting rice fields in Kyoto, Japan. Copeia 4:940-947.

Hirai, T. y M. Matsui. 2002. Feeding relationships between Hyla japonica and Rana nigromaculata in rice fields of Japan. Journal of Herpetology 36:662-667.

Hurtubia, J. 1973. Trophic diversity measurement in sympatric predatory species. Ecology 54:991-999.

Lajmanovich, R. C. 1996. Dinámica trófica de juveniles de 
Leptodactylus ocellatus (Amphibia: Anura), en una isla del Paraná, Argentina. Cuadernos de Herpetología 10:11-23.

Levins, R. 1968. Evolution in changing environments. Princeton University Press, New Jersey 122 p.

Levings, S. C. 1983. Seasonal, annual and among site variation in the floor ant community of a tropical deciduous forest: some causes of patchy species distributions. Ecological Monographs 53:435-455.

López, J. A, M. M. Arias, P. Peltzer y R. C. Lajmanovich. 2005a. Dieta y variación morfométrica de Leptodactylus ocellatus (Linneaus, 1758) (Anura: Leptodactylidae) en tres localidades del centro este de Argentina. Boletín de la Asociación Herpetológica Española 16:32-38.

López, J. A., P. M. Peltzer y R. C. Lajmanovich. 2005b. Dieta y solapamiento del subnicho trófico de nueve especies de leptodactilidos en el Parque General San Martín (Argentina). Boletín de la Asociación Herpetológica Española 19:19-31.

López, J. A., R. Ghirardi, P. A. Scarabotti y M. C. Medrano. 2007. Feeding ecology of Elachistocleis bicolor in a riparian locality of the middle Paraná River. Herpetological Journal 17:48-53.

López, J. A., P. A. Scarabotti, M. C. Medrano y R. Ghirardi. 2009. Is red spotted green frog (Hypsiboas punctatus, Anura: Hylidae) selecting its preys? The importance of prey availability. International Journal of Tropical Biology 57:847-857.

López, J. A., P. A. Scarabotti y R. Ghirardi. 2011. Seasonal patterns of abundance and recruitment in an amphibian assemblage from the Middle Paraná River floodplain. Interciencia 36:538-544.
Neiff, J. J. 1996. Large rivers of South America: toward the new approach. Verhandlungen des Internationalen Verein Limnologie 26:167-180.

Peltzer, P. M. y R. C. Lajmanovich. 2001. Habitat fragmentation and amphibian species richness in riparian areas of the Paraná River, Argentina. Froglog 46:5.

Peltzer, P. M., A. M. Attademo, R. C. Lajmanovich, C. M. Junges, A. H. Beltzer y L. C. Sánchez. 2010. Trophic dynamics of three sympatric anuran species in a soybean agroecosystem from Santa Fe Province, Argentina. Herpetological Journal 20:261-269.

Pianka, E. R. 1974. Niche overlap and diffuse competition. Proceedings of the National Academy of Sciences 71:21412145.

Pielou, E. C. 1969. An introduction to mathematical ecology. Wiley, Hoboken, New Jersey 286 p.

Pyke, G. H. 1984. Optimal foraging theory: a critical review. Annual Review of Ecology and Systematics 15:523-575.

Quiroga, L. B., E. A. Sanabria y J. C. Acosta. 2009. Size- and sexdependent variation in diet of Rhinella arenarum (Anura: Bufonidae) in a wetland of San Juan, Argentina. Journal of Herpetology 43:311-317.

Schoener, T. W. 1974. Resource partitioning in ecological communities. Science 189:27-39.

Wells, K. D. 2007. The ecology and behavior of amphibians. The University of Chicago Press, Chicago. 1148 p.

Williams, Y., S. E. Williams, R. A. Alford, M. Waycott y C. N. Johnson. 2006. Niche breath and geographical range: ecological compensation for geographical rarity in rainforest frogs. Biological letters 2:532-535. 